\title{
Aplicación del modelo QFD en dispositivos de almacenamiento personalizados para mejorar su diseño e impacto en el mercado
}

\author{
Marco Santiago Chiluisa Candelejo \\ mschiluisac@uce.edu.ec \\ Universidad Central del Ecuador, Ecuador \\ Christian Leonardo Chimbo Naranjo \\ chchina@doctor.upv.es \\ Universidad Politécnica de Valencia, España
}

\section{RESUMEN}

El Modelo de Despliegue de Calidad (QFD) es una herramienta muy utilizada para el diseño tanto de productos como de servicios, que permite identificar los criterios más importantes para el cliente o usuarios del producto y / o servicio. Este artículo fue elaborado con el objetivo de desarrollar el QFD para identificar aquellos requisitos de los usuarios en sistemas de almacenamiento industrial (RACK) y utilizar los resultados como principios necesarios, a considerar establecer para ofrecer la mejor opción que satisfaga las necesidades y requerimientos del cliente todos basados, prioritariamente, en el mercado actual y sus demandas en un servicio personalizado. La opinión de los usuarios se derivó de encuestas que consideran la competencia y el mercado actual. Se concluye que el QFD favorece el conocimiento detallado de las necesidades de los usuarios y el análisis de las características técnicas para satisfacer esas necesidades.

Palabras clave: QFD; Sistemas de Almacenamiento; requerimientos del cliente; requerimientos técnicos; Racks. 
Aplicación del modelo QFD en...

\title{
Application of the QFD model in customized storage devices to improve their design and market impact.
}

\begin{abstract}
The Quality Deployment Model (QFD) is a widely used tool for the design of both products and services, which allows to identify the most important criteria for the customer or users of the product and / or service. This article was prepared with the objective of developing the QFD to identify those requirements of users in industrial storage systems (RACK) and use the results as necessary principles, to consider establishing to offer the best option that meets the needs and requirements of the customer all based, primarily, on the current market and its demands in a personalized service. The users' opinion was derived from surveys that consider the competition and the current market. It is concluded that QFD favors detailed knowledge of user needs and analysis of the technical characteristics to meet those needs.
\end{abstract}

Key words: QFD, storage systems; customer requirements; technical requirements; racks.

Artículo recibido: 05 octubre. 2021 Aceptado para publicación: 02 noviembre 2021 Correspondencia: mschiluisac@uce.edu.ec Conflictos de Interés: Ninguna que declarar 


\section{INTRODUCCIÓN}

La calidad, el costo y el tiempo de desarrollo de un servicio tienen un gran impacto directo en la productividad, la participación de mercado y la rentabilidad de una empresa. A medida que los usuarios se vuelven más conscientes de sus necesidades, es importante un mayor nivel de calidad y un tiempo de respuesta más rápido. Es decir, si desea ganar y mantener participación de mercado, debe identificar los requisitos y necesidades antes de competir con el producto o servicio que desea el cliente. De tal modo que el balance y optimización que se logre entre calidad, costo y tiempo depende la participación dentro del mercado.

Por lo tanto, es importante implementar metodologías como el Despliegue de la función calidad, que permitan una identificación temprana y objetiva de las necesidades del cliente (Luis D , 2019).

Actualmente, esta herramienta es muy utilizada en los fabricantes de automóviles, entre ellos Toyota Motor Corporation, que menciona su gran ventaja en el desarrollo de un vehículo, ya que ha permitido un $60 \%$ de ahorro de costos, así como un importante ahorro de tiempo en el desarrollo (F \& RICHARD B, 2014).

La metodología QFD nos permite generar una visión objetiva de lo que buscan los usuarios en un producto / servicio y los requisitos que deben tener, además nos permite priorizar qué funcionalidades son las más importantes para agregar y cuáles no son necesarias. Nos ayuda a determinar la situación de cómo nuestro producto / servicio actual se compara con la competencia, y qué aspectos mejorar para ser más competitivos (Romero, 2019).

El propósito de este artículo es presentar la aplicación de la metodología QFD el cual nos permita desarrollar planes de acción a partir de un diagnóstico con el fin de ofrecer la mejor opción en la implementación de Sistemas de Almacenamiento industrial (Racks) ver en la Figura 1, que satisfagan las necesidades y requerimientos del cliente todo ello basándose como prioridad el mercado actual cuyas demandas son un servicio y diseño personalizado, el estudio se llevará a cabo dentro de una empresa ubicada en Ecuador-Quito que brinda servicios en asesoría, fabricación e implementación de dispositivos de almacenamiento industrial personalizados. 
Figura 1.Sistemas de Almacenamiento Industrial (Racks)

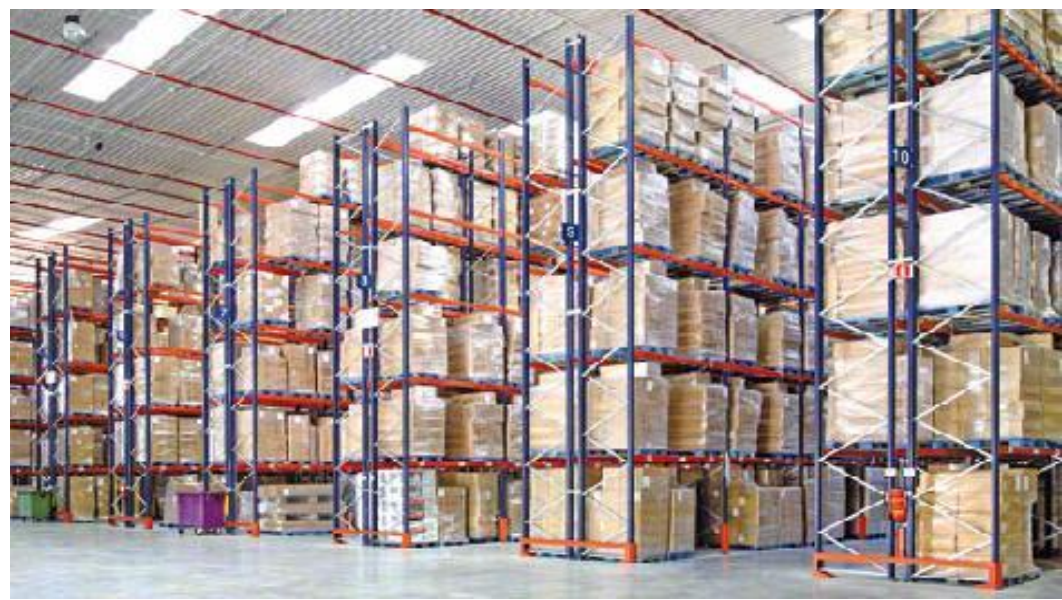

\section{MÉTODOS}

El estudio se lo elaboro en una empresa Metalmecánica que se encarga de la asesoría, diseño, fabricación e implementación de sistemas de almacenamiento industrial (Racks) todo aquello con el fin de brindar al público soluciones integrales en cada una de las áreas anteriormente mencionadas.

De tal modo es necesario contemplar el desarrollo de la Casa de la Calidad ver figura 2:

Figura 2. Casa de la Calidad QFD (Herrera \& Molina, 2019)

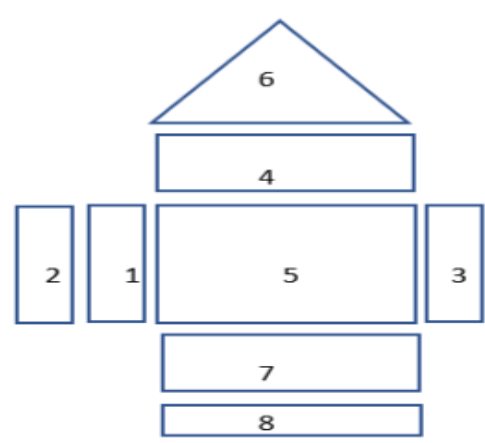

\footnotetext{
1.-Requerimientos del Cliente (RC)

2.-Factor de importancia del cliente

3.-Evaluacion competitiva del cliente

4.-Requerimientos técnicos (RT)

5.-Matriz de Relaciones

6.-Correlacion entre los requerimientos

técnicos

7.-Evaluacion competitiva técnica

8.-Puntuacion
}

El desarrollo de la Casa de la Calidad se ha implementado de la siguiente manera:

\section{Paso I: Investigación de la Voz del Cliente establecimiento de la lista de requerimientos del cliente y el factor de importancia de cada uno}

\section{Fase Cualitativa}

Para la Investigación de la Voz del cliente se procede a realizar una encuesta a clientes en Sistemas de Almacenamiento Industrial (Racks), de tal manera es primordial la toma de una muestra y se consideró los siguientes parámetros (Gamboa, 2018): 
- Tamaño de la población :57

- Nivel de Confianza :95\%

- Margen de error:10 \%

Con estos parámetros, se obtuvo que el tamaño de la muestra debe ser de 37 clientes a quienes se aplicara una encuesta en donde indicaran cuáles son sus requerimientos y/o necesidades.

\section{Fase Cuantitativa}

Una vez efectuada la encuesta a clientes en Sistemas de almacenamiento, en función a la muestra obtenida se procede a identificar y establecer la importancia de cada uno de ellos la misma que constara con escalas de 1 al 5 considerando a 5 como el requerimiento más importante para la decisión o adquisición del producto (Villacis, 2019).

También se requiere realizar una Evaluación Competitiva, requiere una recopilación de información sobre el nivel de cumplimiento de los requerimientos del cliente en los productos existentes en el mercado. Esta información sirve como una referencia comparativa en relación con los posibles competidores más fuertes. Este muestreo se puede realizar a través de encuestas de opinión (F \& RICHARD B, 2014). Para este fin es necesario que los clientes o usuarios cuenten con pautas de como efectuar la evaluación en función del grado de satisfacción obtenidos con el uso del producto, para este fin se establece una escala de calificación en donde 1 representa (muy malo) y 5 para (muy bueno).

Se realizó una relación entre las empresas existentes en el mercado para el diseño y fabricación de los sistemas, con el fin de comparar las ventajas de cada uno de ellos. A continuación, se describen las características de cada una de ellas en la fabricación de los Sistemas de Almacenamiento Industrial (Racks).

- Nosotros: Es una empresa del sector de la metal mecánica que se especializa en sistemas de exhibición y almacenaje en sus partes y accesorios. Su equipo tiene más de 35 años de experiencia en brindar sistemas de almacenamiento industrial de alta calidad (ARTIALAMBRE, 2021).

- Competencia 1: Esta empresa nacional fue fundada en 1971 ofreciendo al mercado productos, que satisfacen las necesidades de almacenamiento y exhibición en 2005 se estableció como una sociedad de responsabilidad limitada, con el tiempo ha fortalecido 
su imagen y posición dentro del mercado, compitiendo con multinacionales. Posee un talento humano integrado, cohesionado, capacitado y dirigido por los diferentes procesos, garantizando la calidad del producto enfocado a la investigación y desarrollo le ha permitido obtener la certificación de calidad ISO 9001 versión 2015 (DUKEMATRIZ, 2021).

- Competencia 2: Es una empresa enfocada al sector comercial, industrial y del hogar, que se especializa en el diseño, desarrollo, fabricación e instalación de estructuras para exhibición y almacenamiento. Esta empresa del sector industrial creada en el año 2016 ha ido creciendo e implementando herramientas y procesos en las distintas áreas tanto comercial, diseño y manufactura logrando ganar acogida en el mercado Nacional una de esas mejoras ha sido la implementación de herramientas CAD como Inventor la cual permite la documentación y simulación de productos permitiendo el trabajo de manera paramétrico en los diseños además de proporcionar herramientas para la manufactura e interpretación en maquinaria CNC (AUTODESK, 2021) (RACKS DEL PACIFICO, 2021).

\section{Paso II: Traducción de la voz del cliente (Ques) en acciones (Comos)}

Una vez que se tiene identificado la lista de requerimientos se requiere una traducción de aquellos requerimientos en características de diseño, del proceso a estos se los identificara como "Comos" estos deberán cumplir las siguientes características (Herrera \& Molina, 2019);

- Estar en Términos Cuantificables

- Reflejar las necesidades del cliente

Se efectúa una valoración que tienen los Requerimientos del Cliente a partir de una escala de relación denominada clásica japonesa en la cual se designa a cada símbolo un valor determinado.

Relación fuerte: $(\bullet)$, equivale al número 9.

Relación moderada: $(\mathrm{O})$, equivale al número 3.

Relación débil o baja: $(\nabla)$, equivale al número 1 .

Cuando no existe relación se deja el espacio vacío y equivale al número 0 (Herrera \& Molina, 2019). 


\section{Paso III: Definición de Objetivos de Diseño (Cuantos)}

Una vez que se han establecido los Como's, es necesario establecer los "Cuantos" estos representan el nivel de desempeño en el que se debe realizar cada uno de los "Como", es apropiado que cada uno de los "Como" tenga al menos un " Cuánto "y representan el desempeño de cada uno de los “Como" además estos deben ser cuantificables (Herrera de la Barrera \& Narvaez Zuñiga, 2017).

\section{Paso IV: Definición de la dificultad Organizacional}

Antes de emprender una acción de "cómo", se debe evaluar la complejidad de su implementación dentro de la organización. Esto se hace utilizando una matriz que tiene en cuenta varios criterios y ponderaciones numéricas ver Tabla 1 (F \& RICHARD B, 2014) :

Tabla 1.Matriz Dificultad Organizacional

\begin{tabular}{lllllll}
\hline Criterio & \multicolumn{4}{c}{ DIFICULTAD } & DO Asignado \\
& $\mathbf{1}$ & $\mathbf{2}$ & $\mathbf{3}$ & $\mathbf{4}$ & $\mathbf{5}$ & \\
\hline Tiempo & & & & \\
Necesario & \\
Recursos \\
Económicos \\
Recursos \\
Humanos \\
\hline Matriz aplicada para Definición de Dificultad Organizacional
\end{tabular}

\section{Paso V: Definición de la Posición competitiva técnica}

Es necesario realizar un análisis de desempeño tomando en cuenta el grado de cumplimiento de los requerimientos del cliente, además, es recomendable conocer y determinar el desempeño de la competencia evaluando los mismos productos (López, Aguilar, \& Jimenéz, 2016).

Esta valoración permitirá determinar un valor de los objetivos a ser alcanzados y resaltar aquellos que sean más competitivos para que de esta manera se los pueda implementar en el producto a diseñar (Aguilar-Zambrano, 2013).

\section{Paso VI: Ponderación de las Acciones (Comos)}

Se requiere efectuar una evaluación basada en la combinación de las relaciones de la importancia técnica $w_{j}$, que es una combinación de la relación $r_{i j}$, de los 
Requerimientos del Cliente y los Requerimientos Técnicos y el factor de importancia de los requerimientos del cliente $d_{i}$ (Romero, 2019).

$w_{j}=\sum_{i=1}^{m} d_{i} \times r_{i j}$

donde:

$w_{j}=$ importancia técnica;

$r_{i, j}=$ relación entre los $\mathrm{RC}$ y los $\mathrm{RT}$;

$d_{i}=$ importancia de los requerimientos del cliente;

$i=1,2, \ldots, m$; siendo $m$ el número de requerimientos del cliente;

$j=1,2, \ldots, n$; siendo $n$ el número de requerimientos técnicos.

Teniendo en cuenta que los valores obtenidos en la evaluación no tienen una interpretación física se los puede emplear para decidir cuales RT serán prioridad y en cierta menar deberían recibir una mayor asignación de recursos. De esta manera debajo de cada valor de importancia técnica se puede obtener un nivel jerárquico o número de prioridad de cada requerimiento técnico (F \& RICHARD B, 2014).

\section{Paso VII: Identificación de Correlaciones}

Para definir la correlación entre los Requerimientos Técnicos se toma como referencia el panel triangular de la parte superior de la casa de la calidad, la misma que se le denomina Techo para el desarrollo de nuevos productos es necesario conocer el efecto que existe en una mejora. Este tipo de información es crítica además es fundamental para de cierta manera satisfacer los objetivos en conflicto (BOSSERT, 1991) (VEZZETI, 2008).

Las figura (@) significa una correlación fuertemente positiva, la figura $(O)$ indica una correlación positiva, la figura $(\&)$ indica una correlación negativa y la figura (\# ) una correlación fuertemente negativa.

\section{Paso VIII: Diagnostico}

Par un correcto Diagnostico se deberá considerar los siguientes Criterios (Romero, 2019) ver Tabla 2 : 
Tabla 2.Criterios de Diagnostico

\begin{tabular}{|c|c|}
\hline Diagnostico & Identificación \\
\hline Punto Critico & $\begin{array}{l}\text { Requerimiento muy importante, y la } \\
\text { evaluación competitiva técnica muestran } \\
\text { un área real de oportunidad para la } \\
\text { empresa. }\end{array}$ \\
\hline Conflicto & $\begin{array}{l}\text { Requerimiento muy importante, a través } \\
\text { de la evaluación cuantitativa, difiere de } \\
\text { nuestro concepto o percepción del } \\
\text { producto y/o servicio. }\end{array}$ \\
\hline Importancia Técnica & $\begin{array}{l}\text { Consiste en identificar los CÓMOS de } \\
\text { mayor peso, ya sea absoluto o relativo. }\end{array}$ \\
\hline Ventaja Competitiva & $\begin{array}{llll}\text { La opinión del cliente, sobre } & \text { el } \\
\text { requerimiento con un grado } & \text { de } \\
\text { importancia alto, es excelente. } & \text { Es } \\
\text { necesario mantener y dar a conocer. } & \end{array}$ \\
\hline Área de oportunidad & $\begin{array}{l}\text { Todos los competidores son calificados } \\
\text { muy mal por el cliente, en un } \\
\text { requerimiento muy importante. }\end{array}$ \\
\hline Indispensable Mejorar & $\begin{array}{l}\text { Si no se puede sobresalir, la premisa es al } \\
\text { menos imitar. }\end{array}$ \\
\hline Evaluación Pobre & $\begin{array}{l}\text { Requerimiento pobre y es de poca } \\
\text { importancia. No se debe ejercer ninguna } \\
\text { acción solo vigilar. }\end{array}$ \\
\hline
\end{tabular}

Fuente: Criterios Aplicados para el Diagnostico

\section{Paso IX: Matriz de Priorización}

Una vez realizado el diagnóstico, se crea una matriz de priorización de acciones, para ello se desarrolla una rúbrica que toma en cuenta varios criterios, ver Tabla 3.

Tabla 3.Matriz de Priorización

\begin{tabular}{|c|c|c|c|c|}
\hline Categoría & Calificación & & Criterio & Descripción \\
\hline \multirow{3}{*}{ Impacto } & 1 & $\begin{array}{c}\text { Poco } \\
\text { Impacto }\end{array}$ & $\begin{array}{l}\text { Acción que genera } \\
\text { poco impacto que solo } \\
\text { permiten atacar y } \\
\text { solucionar un (Como) } \\
\text { establecido. }\end{array}$ & $\begin{array}{lr}\text { Acciones } & \text { que } \\
\text { permiten } & \text { generar } \\
\text { un Impacto en la } \\
\text { Calidad } \\
\text { producto }\end{array}$ \\
\hline & 2 & $\begin{array}{l}\text { Medio } \\
\text { Impacto }\end{array}$ & $\begin{array}{l}\text { Acción que genera un } \\
\text { impacto y que ataca a } \\
\text { más de } 1 \text { (Como). }\end{array}$ & $\begin{array}{l}\text { servicio } \\
\text { basándose en } \\
\text { función a un }\end{array}$ \\
\hline & 3 & $\begin{array}{c}\text { Alto } \\
\text { Impacto }\end{array}$ & $\begin{array}{l}\text { Acción que genera un } \\
\text { alto impacto y que }\end{array}$ & $\begin{array}{l}\text { diagnóstico } \\
\text { situacional }\end{array}$ \\
\hline
\end{tabular}




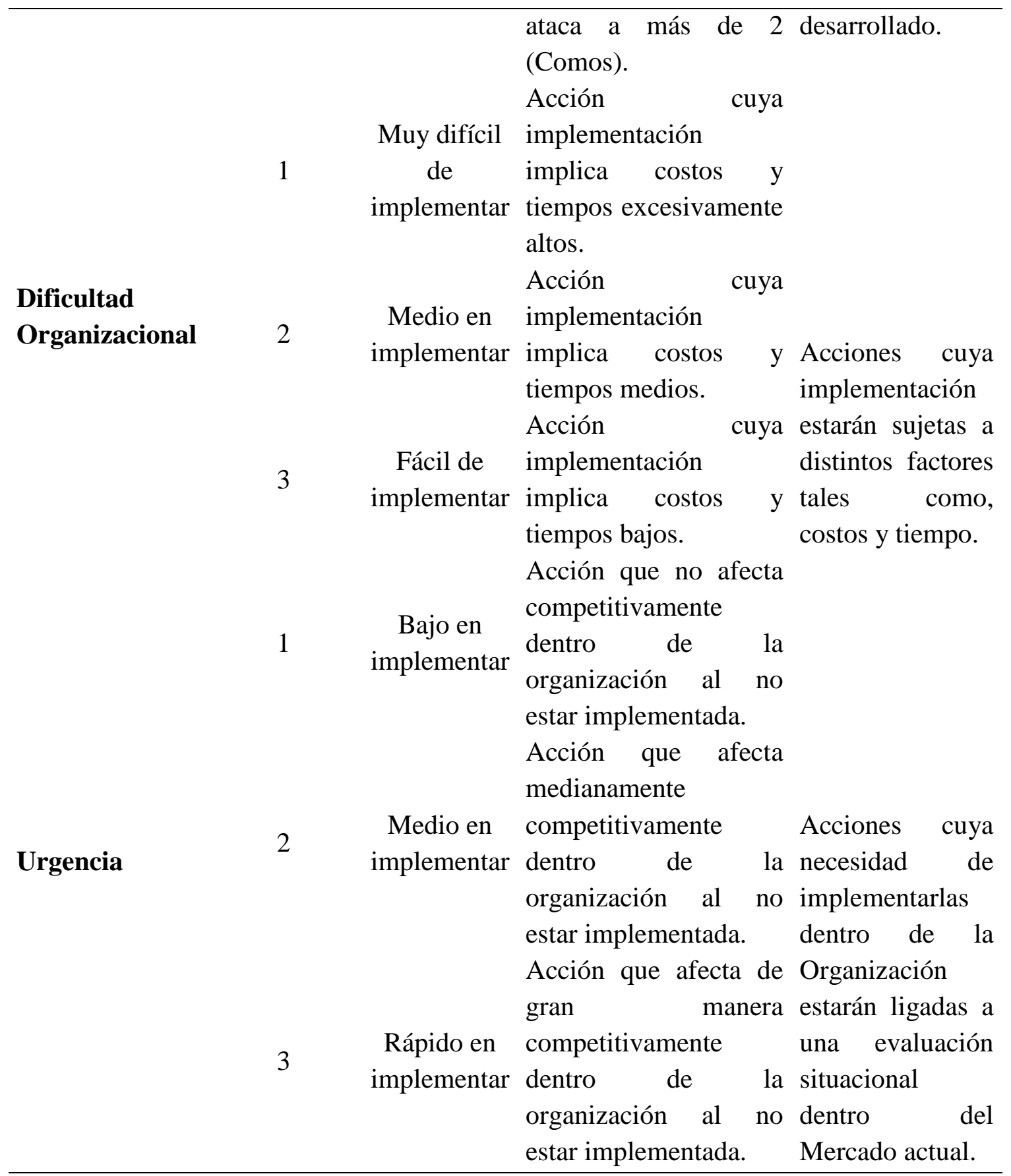

Fuente: Criterios Aplicados para la Priorización de Acciones

\section{Paso X: Planes de Acción}

Para finalizar una vez efectuada la matriz de priorización, se enlista las acciones de menor a mayor dificultad de implementación y se menciona a detalle la implicación, recursos y beneficios de cada una al ser implementadas. 


\section{RESULTADOS Y DISCUSIÓN}

\section{Requerimientos del Cliente y su factor de importancia}

A partir de una encuesta de satisfacción a clientes cuya necesidad son Sistemas de Almacenamiento Industrial se logró definir los requerimientos cada uno con su correspondiente categorización y ponderación designada por los clientes, como muestra la Tabla 4.

Tabla 4.Requerimientos del cliente con su factor de Importancia

\begin{tabular}{ll}
\hline Requerimiento del cliente & Factor de importancia $\left(d_{i}\right)$ \\
\hline 1.-Que optimicen el espacio para almacenar & 5 \\
2.-Que se acoplen al producto a almacenar o exhibir & 5 \\
3.-Que se adecuen a la operación de la bodega & 5 \\
4.-Que se acople al espacio que se disponen & 3 \\
5.-Que sean fáciles de transportar e instalar & 5 \\
6.-Que sean fáciles de diferenciar sus usos & 3 \\
7.-Que soporten la manipulación constante & 4 \\
8.-Que se puedan acoplar a distintas medidas (Modulares) & 5 \\
9.-Que se puedan acoplar accesorios para la identificación & 2 \\
10.-Que estén fabricadas bajos Normas & 3 \\
11.-Que sus materiales sean resistentes & 3 \\
12.-Que soporten la corrosión & 5 \\
13.-Que soporten las cargas de la mercadería & 1 \\
14.-Que tengan seguridades & 5 \\
15.-Que no requieran obras civiles al instalarlas. & 5 \\
\hline
\end{tabular}

Fuente: Resultados de encuestas efectuadas a Clientes en Sistemas de almacenamiento

\section{Industrial}

En la Figura 3 se muestra como las distintas Empresas del sector no cumplen en su totalidad con todas las expectativas de los clientes ya que no integran todas las necesidades al momento de presentar sus servicios al cliente. 
Figura 3.Representación de la evaluación competitiva del cliente

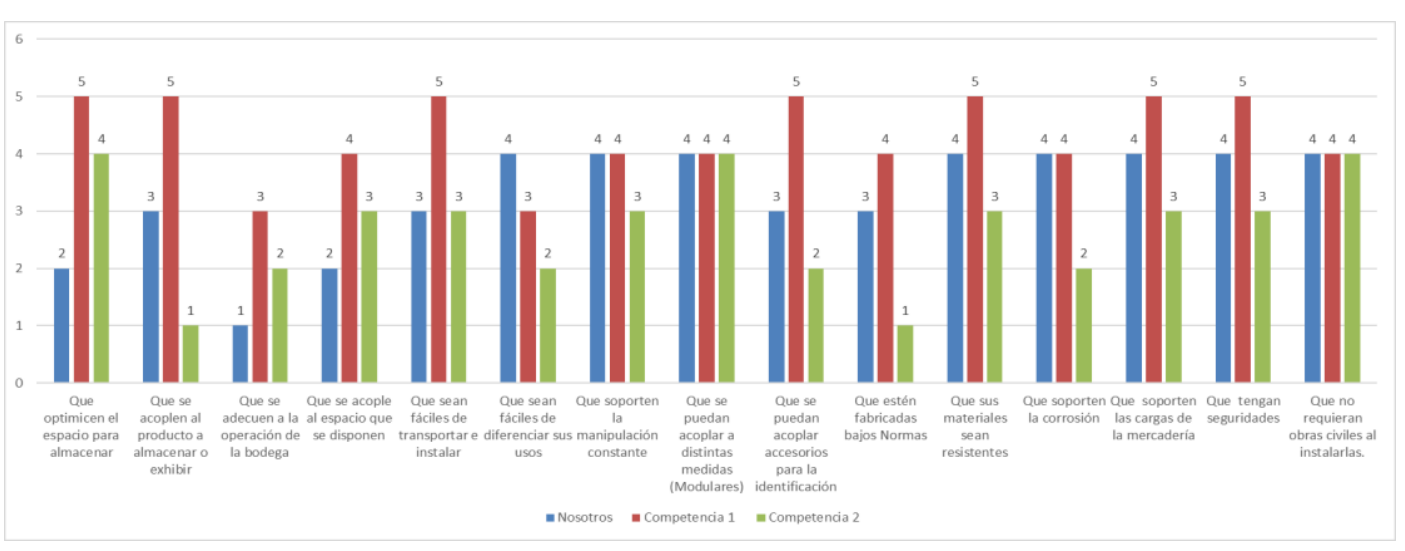

\section{Requerimientos técnicos (Comos-Cuantos)}

Se elaboro la lista de Requerimientos Técnicos (Comos-Cuantos) que se deberá cumplir ver Tabla 5.

Tabla 5. Requerimientos Técnicos (Cómos-Cuantos)

\begin{tabular}{|c|c|}
\hline COMOS & CUANTOS \\
\hline Área en las Planimetrías & Área máxima \\
\hline Análisis de alternativas con simulación & Al menos 2 opciones \\
\hline Medidas de los productos a almacenar & Plano $100 \%$ para simulación \\
\hline Caracterización de la operación de la bodega & Ckecklist $100 \%$ lleno \\
\hline Peso x metro lineal & mínimo posible \\
\hline Identificación & 6 tipos de identificación \\
\hline Manuales y Catálogos & 1 por cada modelo de racks $\%$ \\
\hline Análisis por elementos finitos & 1 por cada proyecto \\
\hline $\begin{array}{l}\text { Sistemas de anclajes al piso por pernos de } \\
\text { anclaje }\end{array}$ & 2 pernos de anclaje por cada parante \\
\hline $\begin{array}{l}\text { Superficie acabada en pintura en polvo } \\
\text { electrostática }\end{array}$ & $\begin{array}{l}\text { Superficie en epoxi-poliéster termo } \\
\text { endurecible en espesor de } 80 \text { micras }\end{array}$ \\
\hline Vinchas de seguridad en las uñetas de las vigas & 1 par por viga \\
\hline Fichas técnicas de Proyectos & 1 por cada proyecto \\
\hline Etiquetado de información en vigas & 1 etiqueta por viga \\
\hline Visitas técnicas en sitio & 3 visitas \\
\hline Asesoramiento técnico & $100 \%$ por cada cliente \\
\hline Sistemas de acoples universales & $\begin{array}{l}\text { piezas } 100 \% \text { compatibles entre los } \\
\text { sistemas }\end{array}$ \\
\hline
\end{tabular}

Fuente: Resultados Obtenidos a partir de la Empresa de estudio 


\section{Dificultad Organizacional}

En la Tabla 6, se muestra el consolidado de la Dificultad Organizacional asignada a cada Como.

Tabla 6.Dificultad Organizacional

\begin{tabular}{ll}
\hline COMOS DO & DOS
\end{tabular}

1.-Área en las Planimetrías

12

2.-Análisis de alternativas con simulación

3.-Medidas de los productos a almacenar

4.-Caracterización de la operación de la bodega

5.-Peso x metro lineal

6.-Identificación

7.-Manuales y Catálogos

8.-Análisis por elementos finitos

9.-Sistemas de anclajes al piso por pernos de anclaje

10.-Superficie acabada en pintura en polvo electrostática

11.-Vinchas de seguridad en las uñetas de las vigas

12.-Fichas técnicas de Proyectos 9

13.-Etiquetado de información en vigas $\quad 7$

14.-Visitas técnicas en sitio 13

15.-Asesoramiento técnico 12

$\begin{array}{ll}\text { 16.-Sistemas de acoples universales } & 10\end{array}$

Fuente: Resultados Obtenidos a partir de la Empresa de estudio

\section{Matriz de Relaciones}

En la Figura 4 se despliega la Casa de la Calidad QFD en la misma se remplazaron la simbología de la Matriz de relaciones por los valores que corresponden en función a la escala clásica japonesa. Se expone los Requerimientos del Cliente con su correspondiente interrelación con los Requerimientos Técnicos anteriormente descritos. 
Figura 4.Casa de la Calidad

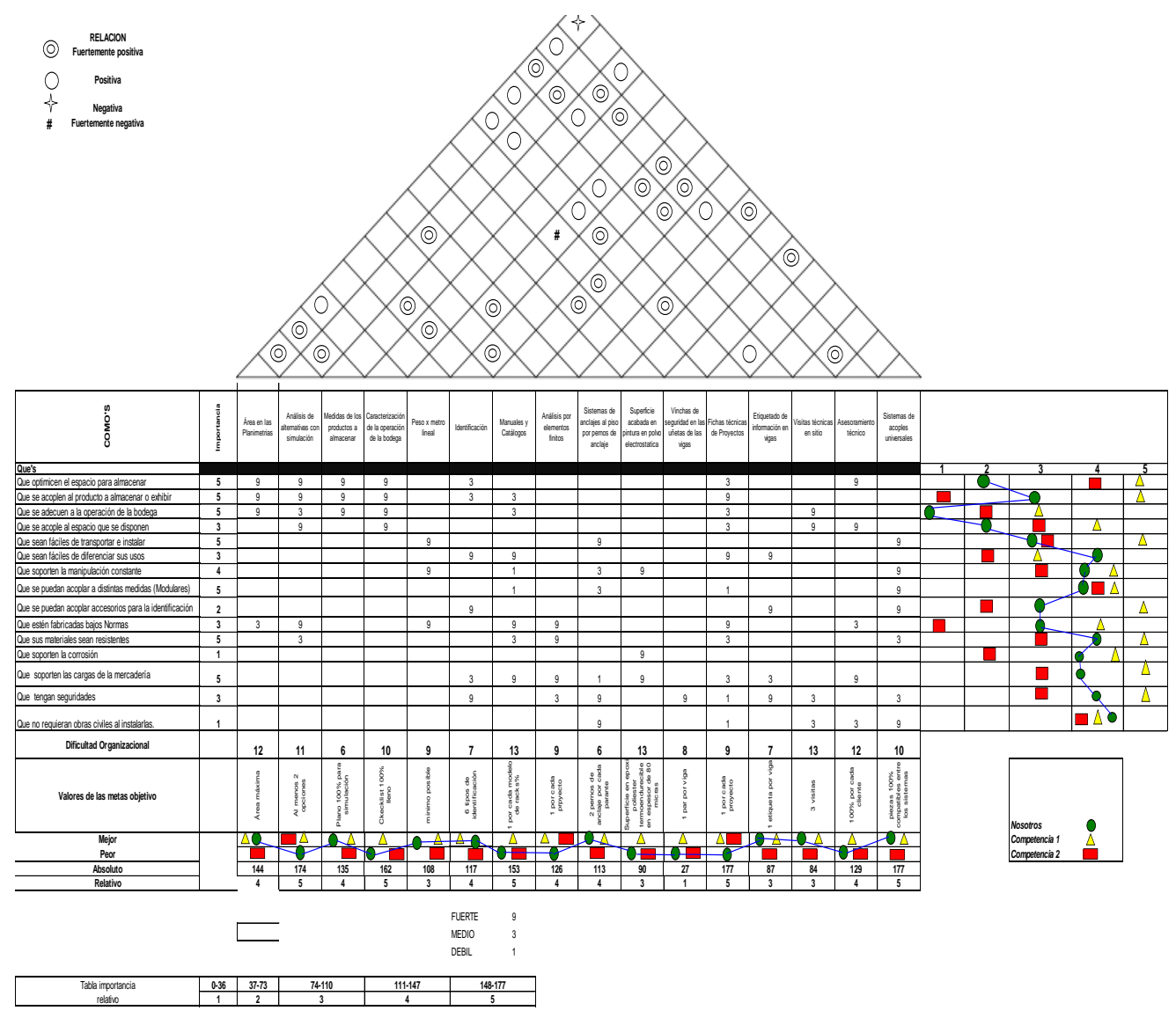

\section{Diagnostico}

Una vez desarrollada en su totalidad la casa de la Calidad QFD se procede a realizar el respectivo diagnostico contemplando los siguientes puntos:

- Punto Crítico. -Que optimicen el espacio para Almacenar y que se adecuen a la operación de la Bodega.

- Conflicto. - Que optimicen el espacio para almacenar, que se adecuen a la operación de la Bodega y que sean fáciles de transportar e instalar.

- Importancia Técnica. - Análisis de alternativas con simulación, Caracterización de la operación de la bodega, Catálogos, Fichas técnicas de proyectos y Sistemas de Acoples Universales.

- Ventaja Competitiva. - La Organización en estudio no presenta ninguna ventaja con respecto a las organizaciones de competencia.

- Área de Oportunidad. - Que se adecuen a la operación de la Bodega.

- Indispensable Mejorar. - Realizar la correcta Caracterización de la Operación de la Bodega. 
- Evaluación Pobre. - Que no requieran obras civiles al instalarlas.

\section{Priorización}

En función a los criterios de Priorización, se enlista las acciones ver en la Tabla 7, de acuerdo con la importancia es decir el que implicaría menos recursos en su implementación.

Tabla 7.Priorización de Acciones

\begin{tabular}{clcccc}
\hline No. & Acciones & Impacto & $\begin{array}{c}\text { Dificultad } \\
\text { Organizacional }\end{array}$ & Urgencia & Importancia \\
\hline 1 & $\begin{array}{l}\text { Caracterización de la } \\
\text { operación de la bodega }\end{array}$ & 3 & 3 & 3 & $\mathbf{9}$ \\
2 & $\begin{array}{l}\text { Medidas de los productos a } \\
\text { almacenar }\end{array}$ & 3 & 2 & 3 & $\mathbf{8}$ \\
3 & $\begin{array}{l}\text { Área en las Planimetrías } \\
4\end{array}$ & 3 & 1 & 3 & $\mathbf{7}$ \\
5 & $\begin{array}{l}\text { Asesoramiento técnico } \\
\text { Análisis de alternativas con }\end{array}$ & 3 & 1 & 2 & $\mathbf{6}$ \\
6 & $\begin{array}{l}\text { Simulación } \\
7\end{array}$ & 3 & 1 & 1 & $\mathbf{5}$ \\
\hline
\end{tabular}

Fuente: Resultados Obtenidos a partir de la Empresa de estudio

\section{Planes de Acción}

\section{Caracterización de la Operación de la Bodega}

Para una correcta Caracterización de la operación de una Bodega, es necesario implementar un formato el cual recopile todos los requisitos de operación de una bodega y otros en el espacio a implementar se deberá describir lo siguiente:

- Dimensiones de Carga a almacenar.

- Peso del producto a almacenar.

- Dimensiones y capacidad de Carga de Montacargas.

- Zonas de Carga y Descarga.

- Levantamiento del espacio disponible (Cerchas y Alturas).

- Tipo de sistema de almacenamiento considerado o planteado para el proyecto.

- Cantidad de Posiciones Pallet que se requieren obtener con el sistema a implementar.

\section{Recursos a Requerir}

- Tiempo: 1 mes

- Humano: 1 persona (1 Diseñador) 
- Inversión: Software de Ofimática -Software de Diseño Gráfico (Ilustrador)

\section{Beneficios}

1. Optimizar el espacio para la implementación de los sistemas

2. Estandariza los parámetros de diseño para la elaboración de propuestas

3. Recoge todas las necesidades del proyecto y del cliente reduciendo con ello que se generen reprocesos y desperdicios al momento de efectuarse la fabricación de los mismos.

\section{Medidas de los Productos a Almacenar}

Para una correcta elaboración de una propuesta, es muy necesario realizar una toma adecuada de las dimensiones del producto o material que estarán sujetos a los distintos sistemas de almacenamiento, a la misma vez esta información recopilada será registrada dentro de un formato que recogerá toda la información correspondiente a la Bodega.

Para lo cual es necesario hacer uso de herramientas de medición que faciliten la toma adecuada de medidas haciendo uso de:

- Flexómetros (Longitud :5 m)

- Calibrador (Apreciación: 0,02 mm)

\section{Recursos a Requerir}

- Tiempo: 1 mes

- Humano: 1 persona (Asesor Técnico Comercial)

- Inversión: Adquisición de Flexómetro y Calibrador

\section{Beneficios}

1. Toma de medidas más exactas de los productos a almacenar.

2. Permite tener un dimensionamiento mucho más real al momento de realizar los diseños de las distintas propuestas.

3. Permite elaborar propuestas económicas mucho más acordes y competitivas en el mercado.

4. Permite mejorar la calidad ya que las propuestas consideran medidas mucho mas exactas.

\section{3. Áreas en Planimetrías}

Con el fin de presentar una propuesta que permite optimizar el espacio, con la mayor cantidad de posiciones pallet es necesario optimizar al máximo las áreas en las 
planimetrías esto se puede lograr a partir de la implementación de un formato para el registro de la información que contiene toda la operación de la bodega (Plan de Acción 1) y con el uso de un software que permita realizar las distribuciones con parámetros reales.

\section{Para lo cual es necesario hacer uso de herramientas de diseño CAD:}

- AutoCAD

\section{Recursos a Requerir}

- Tiempo: 1 mes

- Humano: 1 persona (Diseñador Industrial)

- Inversión: Adquisición de AutoCAD

\section{Beneficios}

1) Reducir tiempos al momento de elaborar propuestas logrando presentar más oportunamente al cliente ganando mayor mercado.

2) Optimizar al máximo la aplicación de los sistemas en los espacios distribuidos logrando satisfacer los requerimientos y necesidades de almacenamiento.

3) Implementar herramienta de diseño 2D-3D que permitan parametrizar cada uno de los espacios y sistemas al momento de elaborar las propuestas.

\section{Asesoramiento Técnico}

Una parte primordial, dentro de todo el proceso de recepción del requerimiento y atención de este es un correcto asesoramiento por parte del personal del área comercial ya que los mismos mantendrán comunicación, directa con el cliente a lo largo de todo el proyecto por tal razón es fundamental fortalecer al personal Comercial esto a partir de las siguientes acciones:

- Capacitación en Logística y Sistemas de Almacenamiento Industrial

- Capacitación interna en el uso correcto de registro de información en los distintos formatos de levantamiento de información (Caracterización de la Operación de la Bodega.

- Capacitación en el uso correcto de herramientas de medición como Flexómetros y Calibradores.

- Capacitación en la interpretación de planos y especificaciones técnicas referentes a cada sistema. 


\section{Recursos a Requerir}

- Tiempo: 1 mes x cada Capacitación

- Humano: Área Comercial (Aproximadamente 3 persona)

- Inversión: Contratación de consultora (Servicios de Logística y Sistemas de Almacenamiento)

\section{Beneficios}

1) Reducir tiempos al momento de brindar una asesoría técnica al cliente.

2) Mejorar la comunicación y satisfacción del cliente a lo largo de la ejecución del proyecto.

3) Ganar mayor acogida dentro del mercado debido a la respuesta inmediata $y$ completa.

4) Facilita el ingreso de la información dentro del Formato de Caracterización de la Operación de la Bodega.

\section{Análisis de Alternativas con Simulación}

Es necesario implementar herramientas de diseño, para el modelado $3 \mathrm{~d}$ y simulación por elementos finitos, con el fin de presentar propuestas mucho más atractivas y acopladas a los requerimientos, por tal razón se propone como herramientas a implementar las siguientes:

- Sketchup (Modelado 3d)

- Inventor o SolidWorks (Modelado Técnico y Simulaciones por elementos finitos)

- AutoCAD (Modelado 2d)

\section{Recursos a Requerir}

- Tiempo: 3 meses

- Humano: 2 (Diseñadores Industriales)

- Inversión: Adquisición de Licencias de los programas (Sketchup-Autocad-Inventor o SolidWorks)

\section{Beneficios}

1) Presentar propuestas mucho más atractivas, acorde al requerimiento del cliente.

2) Estudio mucho más técnico, permitiendo elaborar sistemas mucho más a medida en función a las necesidades específicas del cliente.

3) Tecnificación de la operación de fabricación, de los sistemas ya que permite generar planos e informes de cada proyecto nuevo en desarrollado. 


\section{Fichas Técnicas de Proyectos}

Es necesario mantener un historial, de todos los proyectos como los son las fichas Técnicas es decir los planos y especificaciones correspondientes de los proyectos ejecutados, todo esto con el fin de mantener registrada la información y las distintas opciones desarrolladas en cada uno reduciendo así el tiempo en la elaboración de nuevas propuestas.

\section{Recursos a Requerir}

- Tiempo: 9 meses

- Humano: 1 (Asistente Administrativa)

- Inversión: Repositorio Digital (Nube)

\section{Beneficios}

1) Optimización de recursos en el desarrollo de un nuevo sistema.

2) Fuentes de investigación para la obtención de información para los nuevos proyectos.

\section{Catálogos}

Es primordial implementar herramientas tanto para el área comercial, las mismas que recopilen toda la información general y especifica de cada uno de los sistemas así también que especifiquen la forma de instalación e implementación de los mismos todo ello se lo puede realizar a partir de la implementación de manuales y catálogos los mismos que deben presentar la siguiente información:

- Fotografías de cada sistema y sus características

- Fotografías de proyectos implementados

- Despiece de cada sistema y la aplicación de estos

- Instructivo de armado e instalación de cada uno.

\section{Recursos a Requerir}

- Tiempo: 4 meses

- Humano: 3 (Diseñador Gráfico-Diseñador Industrial-Asesor Técnico Comercial)

- Inversión: Manuales y Catálogos Digitales (Diseñado en conjunto en asesoría de una Agencia Publicitaria-Repositorio Digital).

\section{Beneficios}

1) Brindar una herramienta de trabajo al área comercial e instalación.

2) Ganar mayor acogida en el mercado 


\section{CONCLUSIÓN O CONSIDERACIONES FINALES}

1. Este estudio permitió presentar la experiencia obtenida al momento de aplicar la metodología QFD en cada una de sus distintas fases.

2. Confirió un gran conocimiento sobre la competencia y las cualidades que cada una de ellas dispone dentro del mercado.

3. El estudio efectuado permitió establecer todos los requerimientos concretos a satisfacer del cliente permitiendo con ello establecer un parámetro de pasos a ejecutar.

4. Se pudo constatar como importancia técnica la necesidad de optimizar el espacio para el almacenamiento, así como que todo sistema se pueda acoplar a las dimensiones del producto a almacenar o exhibir también se pudo constatar la necesidad de implementar Catálogos de los sistemas mencionando en ellos las cargas a las que pueden estar sujetos cada uno.

5. Se pudo constatar que como oportunidades de mejora toda propuesta de implementación debe acoplarse a la operación de la bodega.

6. Se pudo constatar que es indispensable la caracterización correcta de la operación de la bodega ya que la misma indica parámetros como pasillos, carga, dimensiones de montacargas zonas de carga y descarga así también como zonas de picking.

7. Las acciones que se sugieren necesarias a implementar de acuerdo a la dificultad organizacional fueron las siguientes:

- Elaboración de un formato que permita la Caracterización de la Operación de la Bodega

- Uso de herramientas de medición como Flexómetros y Calibradores

- Implementación de herramientas para diseño Cad 2D

- Capacitación al personal del Área Comercial

- Implementación de herramientas para Modelado 3d y simulación por elementos finitos.

- Mantener archivado Fichas técnicas de los Proyectos

- Elaboración de Catálogos

8. Cada una de estas acciones permiten optimizar los tiempos de Diseño desarrollo e implementación del Proyecto considerando las áreas Comercial y Producción logrando garantizar una calidad en cada uno de los sistemas a fabricar. 


\section{LISTA DE REFERENCIAS}

Aguilar-Zambrano. (2013). Método interdisciplinar de análisis de productos de apoyo a personas en situación de discapacidad con el uso sinérgico de la funcion de despliegue de calidad y los procesos analiticos jerarquicos. Ingenieria $y$ Universidad,ISSN, 17.

ARTIALAMBRE. (2021).

ARTIALAMBRE. Obtenido

de https://www.artialambre.com/quienes.html

AUTODESK. (2021). Inventor Software CAD 3D de calidad profesional para diseño e ingeniería de productos. Obtenido de AUTODESK: https://latinoamerica.autodesk.com/products/inventor/overview

BOSSERT, J. L. (1991). Quality function deployment: a practitioner's approach. ASQC QUALITY PRESS MILWAUKEE.

DUKEMATRIZ. (2021). DUKEMATRIZ. Obtenido de https://www.racks.com.ec/es/empresa

F, R., \& RICHARD B, C. (2014). Administracion de Operaciones Produccion y Cadena de Suministros. Mexico: MC GRAW HILL.

Gamboa, M. (2018). Estadística aplicada a la investigación educativa. Dilemas Contemporáneos: Educación, Política y Valores.

Herrera de la Barrera , J., \& Narvaez Zuñiga, C. (2017). Metodología para la comprensión de la voz del cliente en entornos dinamicos utilizando el despliegue de funciones de Calidad (QFD). TEKNOS.

Herrera, G., \& Molina, F. (2019). REVISIÓN BIBLIOGRÁFICA DE LA METODOLOGÍA. Universidad Católica San Pablo, Arequipa.

López, J., Aguilar, J., \& Jimenéz, M. (2016). Despliegue de la Función de Calidad para el Diseño de Limpiador Automático de Rodillo. Conciencia Técnológica.

Luis D , G. (2019). QFD Aplicado a los servicios de una Constructora Boliviana. ACADEMIA.

RACKS DEL PACIFICO. (2021). Nosotros. Obtenido de http://racksdelpacifico.com/Nosotros/index.php

Romero, J. (2019). PROPUESTA DE IMPLEMENTACIÓN DEL MODELO QUALITY PARA EL ANÁLISIS DE LA SATISFACCIÓN DEL CLIENTE CON LOS PRODUCTOS OFERTADOS POR LA EMPRESA JUNGLA 
CIA.LTDA. (Tesis de Maestria). PONTIFICIA UNIVERSIDAD CATÓLICA, Quito.

VEZZETI, E. (2008). Computer aided inspection:design of customer-oriented benchnark for noncontact 3D scanner evaluation. International Journal of Advanced Manufacturing Technology, 1140-1151.

Villacis, A. (2019). Aplicación del Modelo QFD para el Análisis de Satisfacción del cliente.Caso:NUNANDES. Pontificia Universidad Catolica, Quito. 